\title{
Ultrafast laser-induced guided elastic waves in a freestanding aluminum membrane
}

\author{
Hao Zhang $\odot,{ }^{1, *}$ Alessandro Antoncecchi, ${ }^{1,2}$ Stephen Edward $\odot,{ }^{1,3}$ Paul Planken, ${ }^{1,3}$ and Stefan Witte $\odot^{1,2, \uparrow}$ \\ ${ }^{1}$ Advanced Research Center for Nanolithography, Science Park 106, 1098 XG Amsterdam, Netherlands \\ ${ }^{2}$ Vrije Universiteit Amsterdam, De Boelelaan 1105, 1081 HV, Amsterdam, Netherlands \\ ${ }^{3}$ Universiteit van Amsterdam, Science Park 904, 1098 XH Amsterdam, Netherlands
}

(Received 21 September 2020; revised 20 January 2021; accepted 25 January 2021; published 4 February 2021)

\begin{abstract}
Ultrafast laser-induced guided acoustic waves in thin, freely suspended films are important for many applications adopting the laser-ultrasonics technique. These waves show unique dispersion relations, leading to minimal propagation losses and acoustic energy confinement. While this principle has been known, the separation of various physical effects in the formation of measured signals involving these guided acoustic waves has not been clearly elaborated. Here, we present a combined experimental and theoretical study on all-optical excitation and detection of these waves in a thin, freestanding aluminum membrane. The acoustic dynamics is excited and measured by using a femtosecond time-resolved pump-probe technique with controlled probing position, enabling spatially resolved detection. The measured signals are compared with an advanced numerical model that we developed earlier [H. Zhang et al., Phys. Rev. Appl. 13, 014010 (2020)], showing excellent agreement. The combination of experiment and simulation allows us to decode various physical effects in the signal formation, including different acoustic field components. Unknown material properties, such as acoustic attenuation coefficients, and the two complex photoelastic constants are quantitatively retrieved by fitting the measured signals. Furthermore, we provide evidence of nonlinear propagation of the excited guided acoustic waves.
\end{abstract}

DOI: 10.1103/PhysRevB.103.064303

\section{INTRODUCTION}

Laser-induced high-frequency (GHz-THz range) elastic waves (also referred to as acoustic waves or hypersound) in solids are extensively used in both industrial and academic research. In contrast to light, almost all solids are transparent to sound, including many optically opaque media. For this reason, many noninvasive methods for evaluating bulk materials based on laser-induced elastic excitations are established. Recent advanced applications include using elastic excitation for communicating with quantum systems [1-3], imaging buried nanostructures [4,5], quantitative retrieval of phononic and mechanical properties [6,7], subsurface detection of alignment gratings for photolithography [8], and more.

Earlier studies on laser-induced elastic waves were mostly focused on the investigation of one-dimensional propagation of excited longitudinal phonons [9-14], which have yielded remarkable results about the phonon generation mechanism itself. Further progress in the field has enabled the generation and detection of shear phonons [15-17] and surface acoustic waves $[16,18,19]$. Among these more complex types of waves,

\footnotetext{
*h.zhang@arcnl.nl

†S.Witte@arcnl.nl
}

Published by the American Physical Society under the terms of the Creative Commons Attribution 4.0 International license. Further distribution of this work must maintain attribution to the author(s) and the published article's title, journal citation, and DOI.
Lamb waves are attracting increasing attention owing to their unique properties and potential for specialized applications. Lamb waves are guided acoustic wave modes existing in thin and freely suspended films [20]. The propagation behavior of Lamb waves can be used for the nondestructive characterization of thin films. One example is the zero-group-velocity modes, where the group velocity of the Lamb wave vanishes, resulting in acoustic energy localization in the source region [21], offering reduced acoustic losses and enabling local material sensing and testing applications [22,23].

The excitation of Lamb waves requires a component of the particle movement in the direction parallel to the free surface. In laser ultrasonics, this is usually realized by either tightly focusing the excitation laser pulse [21,24] or by employing nanostructured surfaces [25]. The dynamics of phonon evolution is then detected by a delayed probe pulse in a pump-probe configuration. Despite the already established progress on laser generation and detection of Lamb waves, there is still a need for a comprehensive and detailed comparison between the measured signal and an advanced model describing the dynamics over timescales that span orders of magnitude. Furthermore, the role of thermal and acoustic contributions as well as different acoustic field components in the evolution of the signal shape and the evolution of the Lamb waves over propagation distance have not yet been fully investigated. Acoustic attenuation of the Lamb waves is another important factor that is of practical importance, as it limits the interaction distance in various applications.

Here, we present a combined experimental and theoretical study of ultrafast laser-induced elastic waves in a thin, freestanding aluminum membrane. In our experiments, we 
(a)

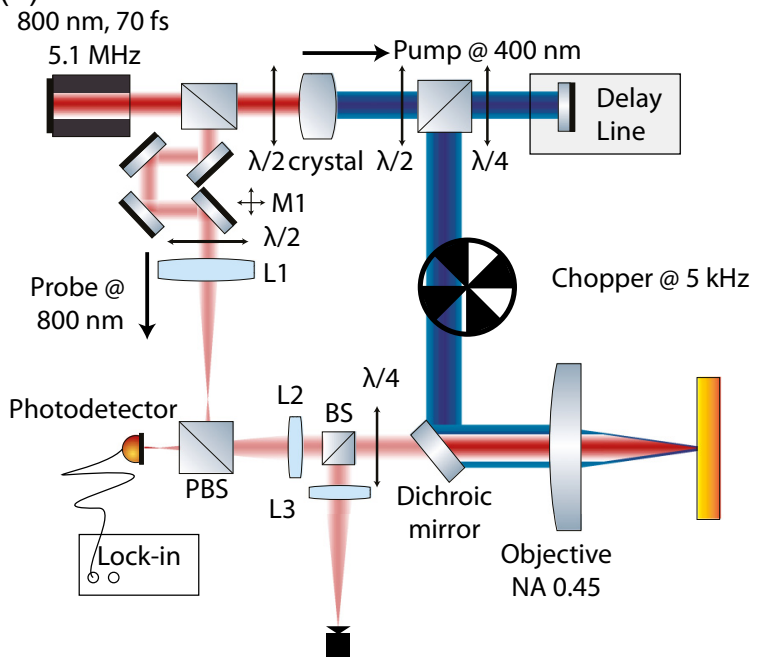

(b) $\times 10^{-3}$

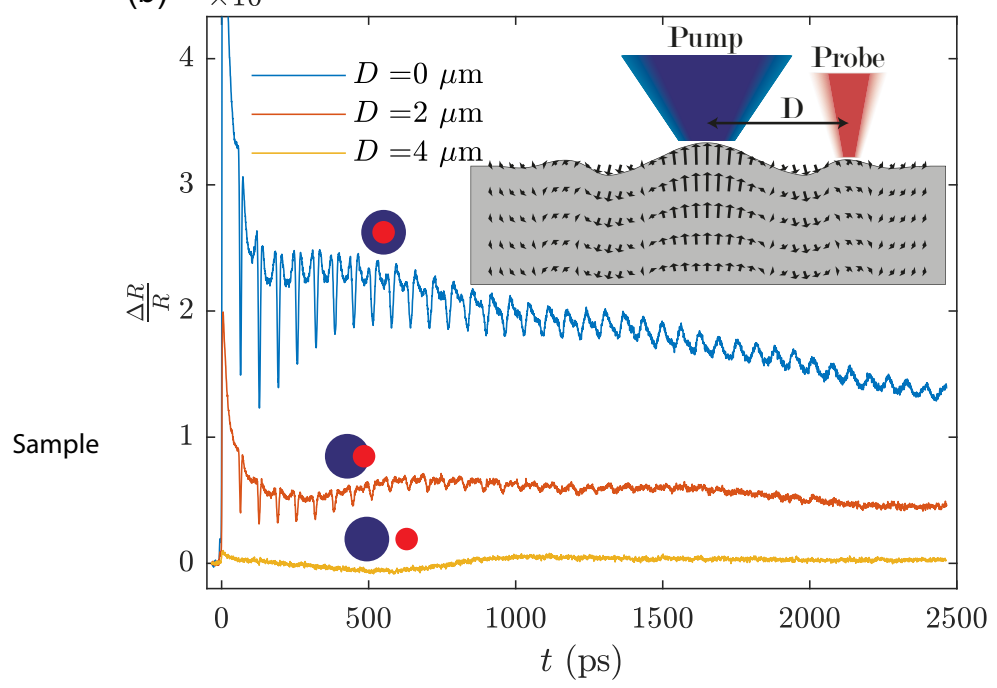

FIG. 1. (a) Schematic of the experimental setup. The pump beam is tightly focused on the sample surface using a microscope objective. The probe beam is positioned at different distances $D$ with respect to the pump beam. (b) Reflectivity changes measured on a $200 \mathrm{~nm}$ aluminum freestanding membrane, for different values of probe shift. The reflectivity changes are measured through a modulation of the dielectric constant via the photoelastic effect [Eq. (2)]. The inset in (b) illustrates the excitation of Lamb waves by the tightly focused pump beam, and its detection using a spatially shifted probe beam. The black arrows are the displacement vector of the excited Lamb waves (not to scale, only for illustrative purpose). The color circles illustrate the relative position of the focused pump (blue) and probe (red) beams for each measurement.

use a tightly focused laser pulse to generate high-frequency Lamb waves. The optical response is probed at various locations with respect to the focused pump pulse. The measured signals are shown to be in excellent agreement with an advanced model that we developed earlier [6]. This combined experimental and theoretical approach allows us to address several key aspects of Lamb wave evolution: the separation of signal contribution due to different strain components, the thermo-optical effects, as well as quantitative determinations of acoustic propagation losses and the complex photoelastic coefficients. In addition, we present evidence of nonlinear propagation of the excited high-amplitude Lamb waves and show that acoustic nonlinearity can alter the measured signal shape.

The paper is organized as follows: In Sec. II, we discuss the experimental setup and the probe position-dependent measurements. In Sec. III, we briefly discuss the model we developed earlier and its adaptation to the current experiment, as well as the fitting approach. In Sec. IV, we present the results and interpretations.

\section{POSITION-DEPENDENT PUMP-PROBE MEASUREMENTS}

A schematic of the experimental setup is shown in Fig. 1(a). The output of a Ti:sapphire oscillator (Femtolasers XL500: wavelength centered at $800 \mathrm{~nm}$, pulse duration $70 \mathrm{fs}$, repetition rate $5.1 \mathrm{MHz}$ ) is split into two beams with a 90:10 beam splitter. The beam with higher intensity is used to generate the second harmonic $(400 \mathrm{~nm})$ of the fundamental wavelength using a $\beta$-barium borate (BBO) crystal. This converted $400 \mathrm{~nm}$ beam is used as the pump beam with a variable optical path length by using a mechanical translation stage (PI) as the optical delay line. This beam is focused by a microscope objective (Olympus LCPLN20XIR, x20, N.A. $0.45)$ onto the sample surface. The pump power after the objective is measured to be $5.5 \mathrm{~mW}$. The beam with the fundamental wavelength $(800 \mathrm{~nm})$ is used as the probe beam. The probe beam is sent through a telescope system consisting of two lenses: L1 with a focal length of $100 \mathrm{~mm}$ and L2 with a focal length of $200 \mathrm{~mm}$. The telescope system expands the beam such that it fills the entire objective aperture to form a diffraction-limited probe spot. The mirror M1 in front of L1 is mounted on a two-axes mirror mount and is imaged onto the back-focal plane of the objective. By changing the angle of incidence of the beam on the lens L1, an area on the sample surface can be scanned by the probe beam, while leaving the pump beam position fixed [26]. We monitor the position of the probe spot on the sample using an imaging system composed of the objective and the L3 $(\mathrm{f}=200 \mathrm{~mm})$ lens which projects an image of the sample plane into a CCD camera. With the camera, we also measure the focal waist of the probe beam as $w_{\text {probe }}=1.2 \mu \mathrm{m}$ at $1 / e^{2}$ intensity. The physical pixel size of the camera is $2.2 \mu \mathrm{m}$, and with a magnification of $20 \mathrm{X}$, we can determine the probe center position with an accuracy close to $100 \mathrm{~nm}$. With the CCD camera, we also measure the focused probe spot size versus the probe displacement. The probe spot size slightly increases as it is moved away from the pump spot. By fitting the probe spot image to a two-dimensional (2D) Gaussian function, the maximum increase in the spot radius is about $100 \mathrm{~nm}$ at the largest probing distance $(5.5 \mu \mathrm{m})$. The size variations at shorter displacements are even smaller. Such small variations of the probe size are far below the spot size itself and estimated lateral acoustic wavelength. Therefore, its effect on the measured signals is negligible. The probe light reflected by the sample is focused on a silicon photodiode (Thorlabs PDA100A2). The photodiode signal is sent to a lock-in amplifier referenced by 
the chopping wheel which modulates the pump beam at a frequency of $5 \mathrm{kHz}$. A calibration of the lock-in output signal allows one to record the absolute reflectivity change.

The reflectivity changes are measured for a range of probing distances $(0-5.5 \mu \mathrm{m})$ on an aluminum freestanding membrane of $200 \mathrm{~nm}$ thickness. The maximum probing distance enables the detection of Lamb waves propagating away from the pumped region. Figure 1(b) shows typical pumpprobe traces measured at three different probing positions. For the zero probing position, a large and sharp peak is observed immediately after the pump excitation, which is caused by an abrupt heating of the free electrons and lattices. This initial peak is not fully shown in the graph to better visualize the acoustic signals that are more important for this work. Repetitive dips are observed after, which corresponds to an acoustic pulse bouncing back and forth in the freestanding membrane. Differences in the background shape are also observed for different probing positions. Detailed discussions and interpretation will be presented in the following sections.

\section{THEORY}

\section{A. The numerical model}

In order to understand the signal formation and to interpret the measured data, we use the numerical model developed earlier in our group [6]. In the model, we incorporate the physics of all the steps involved in a typical picosecond ultrasonics experiment, i.e., the generation, propagation, and detection of the ultrasound waves. For the generation, we treat thermoelasticity as the dominant mechanism in metals $[9,10]$. The evolution of the lattice temperature is modeled by the two-temperature model [27] with actual laser irradiation conditions as model inputs. For the sound propagation, we numerically solve the equation of motion for an isotropic elastic wave, with further incorporation of sound attenuation terms [6,28,29]:

$$
\begin{aligned}
\rho \frac{\partial^{2} \mathbf{u}}{\partial t^{2}}= & \mu \nabla^{2} \mathbf{u}+(\mu+\lambda) \nabla(\nabla \cdot \mathbf{u})+\nabla \sigma_{t h} \\
& +\xi \nabla^{2} \mathbf{v}+\left(\xi+\lambda_{v}\right) \nabla(\nabla \cdot \mathbf{v})
\end{aligned}
$$

where $\sigma_{t h}$ is the thermal stress generated by the laser pulse, $\mathbf{u}$ is the displacement vector, $\rho$ is the mass density, $\lambda$ and $\mu$ are the two Lamé parameters, $\mathbf{v}$ is the velocity vector, $\xi$ is the coefficient of shear viscosity, and $\lambda_{v}$ is linked to the coefficient of bulk viscosity $\eta$ as $\lambda_{v}=\eta-2 \xi / 3$. The thermal stress $\sigma_{t h}$ is coupled to the generation step of the model through thermoelasticity.

Equation (1) is solved by the finite-difference time-domain (FDTD) method [30] in a two-dimensional (2D) geometry with the incorporation of proper boundary conditions. For the front and back surfaces of the membrane, a free boundary condition is implemented which requires the total stress at the air-metal interface to vanish. For the two lateral boundaries, perfectly matched layers (PMLs) [31] are implemented to absorb nonphysical reflections from the edges of the numerical grid. By solving Eq. (1), the two strain components $s_{x}$ and $s_{y}$ as well as the shear strain $s_{x y}$ can be obtained [6]. Here, $x$ refers to the axis perpendicular to the sample surface, while $y$ refers to the axis parallel to it. Having those strain components, the change in the electric permittivity tensor can be evaluated, provided that the photoelastic tensor is known. Here we assume the material is isotropic, and thus the photoelastic tensor has only two unknowns, $P_{11}, P_{12}$ and $P_{44}=\left(P_{11}-P_{12}\right) / 2$ [15]. The probe beam at our photodetector position is linearly polarized, and thus we only have to consider the $y$ component of the electric field of the probe beam. Under these assumptions, the changes in the permittivity tensor elements are

$$
\Delta \epsilon_{y y}=-\epsilon_{r}^{2}\left(P_{11} s_{y}+P_{12} s_{x}\right) ; \quad \Delta \epsilon_{x y}=-\epsilon_{r}^{2} P_{44} s_{x y},
$$

where $\epsilon_{r}$ is the dielectric constant of aluminum in the unperturbed state. In our data analysis, we have found that including $\Delta \epsilon_{x y}$ does not result in a better model fit through the measured data, indicating its negligible contribution. Thus, in the following analysis, $\Delta \epsilon_{x y}=0$.

Having the value of the permittivity change, the reflectivity change can be calculated by solving the Maxwell's equations for the probe pulse. In this work, the transform matrix method [32] is used, which also takes into account the permittivity gradient along the propagation direction of the probe pulse.

\section{B. Least-squares fitting the measured data}

The model described in the previous section is used to fit the measured data using the least-squares algorithm. Material properties such as the electron-phonon coupling constant, thermo-optic coefficient, photoelastic coefficients $P_{11}$ and $P_{12}$, acoustic attenuation coefficient, as well as membrane thickness are treated as fitting parameters. The longitudinal speed of sound in aluminum is fixed as $c_{l}=6420 \mathrm{~m} / \mathrm{s}$ [33] during the fitting. The focal beam waist of the probe beam is measured as $w_{\text {probe }}=1.2 \mu \mathrm{m}$. The measured signal at different probing positions can be seen as a convolution of the probe beam with the response function induced by the pump beam, which already contains information about the size of the focused pump beam. Therefore, the focal beam waist of the pump beam is treated as an extra fitting parameter. Other parameters used in the model are either taken from known literature values or estimated from experimental conditions.

\section{RESULTS AND DISCUSSION}

Measurements were performed on a 200-nm-thick aluminum freestanding membrane for probing distances from 0 to $5.5 \mu \mathrm{m}$ in $0.5 \mu \mathrm{m}$ steps. These values refer to the distances from the center of the focused probe beam to the center of the focused pump beam. The values are determined from the recorded probe spot images during the measurement. In the data analysis, we found that the best match between the measured data and simulations, especially the determined lateral propagation speed of the Lamb waves, was found when a scaling factor of 0.8 was applied to these values of the probing distance. This is likely due to the uncertainty in the determination of the magnification factor of our imaging system, limiting the accuracy of the determination of the probing positions. We therefore applied this scaling to the probing distances to use as both model inputs for fitting the data and the presentation of the final results. We note that the scaling does not change the interpretation of the 


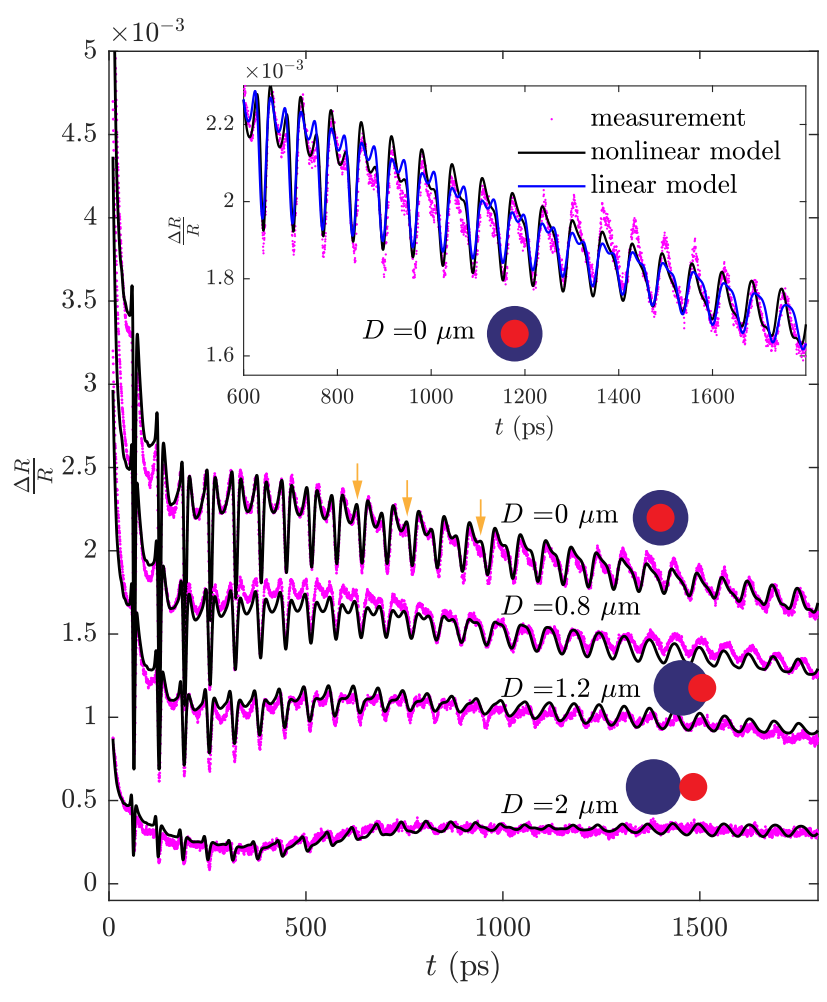

FIG. 2. Measured reflectivity change at four probe positions (magenta dots) and corresponding model fit (black lines) on an aluminum freestanding membrane of $200 \mathrm{~nm}$ thickness. The inset shows the comparison between a linear acoustic model (blue line) and a nonlinear acoustic model (black line).

results. We use the model to fit the measured data at $0,0.8,1.2$, and $2 \mu \mathrm{m}$ probe positions (after applying the scaling factor). The best-fit results are shown in Fig. 2. The agreement with the measured data is excellent. In the fit, we minimize the combined residual of the measurements at these four probing positions. The calculated signals at all probe positions share the same group of best-fit parameters, except for the probing position used for that calculation. The best-fit parameters are shown in Table I. It is worth noting that in Ref. [35], only the ratio between the real part and the imaginary part of the photoelastic constants is given, while our result yields not only the ratio but also the amplitudes of the two photoelastic constants $P_{11}$ and $P_{12}$. This is achieved by calibrating the absolute reflectivity change and by separating different contributions to the measured signals. The measured signals can be well explained by the combined effects of three contributions: the refractive index change induced by the two strain components $s_{x}, s_{y}$ and the thermo-optic effect. In Figs. 3(a)-3(d), we plot the resulting reflectivity changes calculated by each of those contributions alone. The total signal is a coherent sum of the electric-field variations due to the individual contributions, rather than a direct sum of the reflectivities. The signal during the first 50 ps [inset of Fig. 3(a)] is dominated by the combined effects due to the mass density change and the lattice temperature change. The thermal stress introduced by the laser pulse produces a nonpropagating part of the strain component, leading to a rapid increase of the density in the vicinity of the illuminated surface, followed by its slow recovery towards the room-temperature value. This density change is accompanied by the temperature change, which shows the rapid increase and the slowly recovery as well. Although these two effects always accompany each other, they contribute to the measured signal by a different amount through the difference between the photoelastic constant and the thermo-optic coefficient. For probing at $800 \mathrm{~nm}$ wavelength on aluminum, the thermo-optic effect is expected to be large as the probe wavelength coincides with the peak of its thermoreflectance spectrum [38]. The magnitude of these two contributions is quite similar, yet their signal shapes show slight differences: the increase of the thermo-optic contribution is more abrupt, while the strain contribution takes more time to develop. This is to be expected as the strain develops after the temperature increase and requires coherent motion of the lattice. The difference in their signal shapes indicates that separating these two contributions by fitting their combined effect is possible. In fact, excluding the thermo-optic effect in the fit significantly increases the fitting residual. The best-fit thermoreflectance is smaller than the reference value. This may be caused by an overestimation of the incident pump fluence, but another possibility for this discrepancy is cumulative heating due to the high repetition rate of the laser system. This heating increases the steady-state sample temperature, which causes us to extract

TABLE I. Best-fit parameters on $200 \mathrm{~nm} \mathrm{Al} \mathrm{freestanding} \mathrm{membrane.}$

\begin{tabular}{|c|c|c|c|c|}
\hline Parameter & Explanation & Unit & Best-fit value & Reference value \\
\hline$d$ & Membrane thickness (exclude oxide layers) & $\mathrm{nm}$ & 183 & 200 \\
\hline$G$ & Electron-phonon coupling constant & $10^{17} \mathrm{Wm}^{-3} \mathrm{~K}^{-1}$ & 2.92 & $2.46[34]$ \\
\hline$\frac{\partial n}{\partial T}$ & Thermo-optic coefficient, real part & $-1 \times 10^{-3} \mathrm{~K}^{-1}$ & 0.59 & $2.96-4.08^{\mathrm{a}}$ \\
\hline$\frac{\partial \kappa}{\partial T}$ & Thermo-optic coefficient, imaginary part & $10^{-3} \mathrm{~K}^{-1}$ & 0.81 & $0.4-4.4^{\mathrm{a}}$ \\
\hline$w_{\text {pump }}$ & Pump radius $\left(1 / e^{2}\right)$ & $\mu \mathrm{m}$ & 1.8 & not applicable \\
\hline$\frac{d R}{R d T} \mathrm{c}$ & Thermoreflectance at $800 \mathrm{~nm}$ & $10^{-4} \mathrm{~K}^{-1}$ & 0.49 & $1.14-2.4[36-38]$ \\
\hline
\end{tabular}

${ }^{a}$ The reference values on the thermo-optic coefficient are determined by the optical conductivity data at varies temperatures in Ref. [39] together with the available thermoreflectance data in Refs. [36-38].

${ }^{\mathrm{b}}$ In Ref. [35],the amplitude is arbitrary; only the ratio is relevant.

${ }^{\mathrm{c}}$ Calculated from the best-fit thermo-optic coefficient. 
(a)

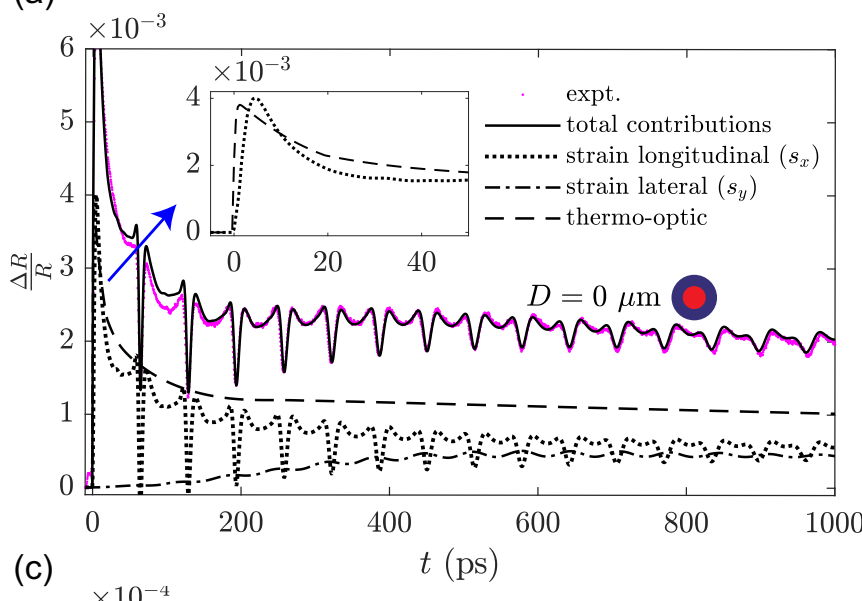

(c)
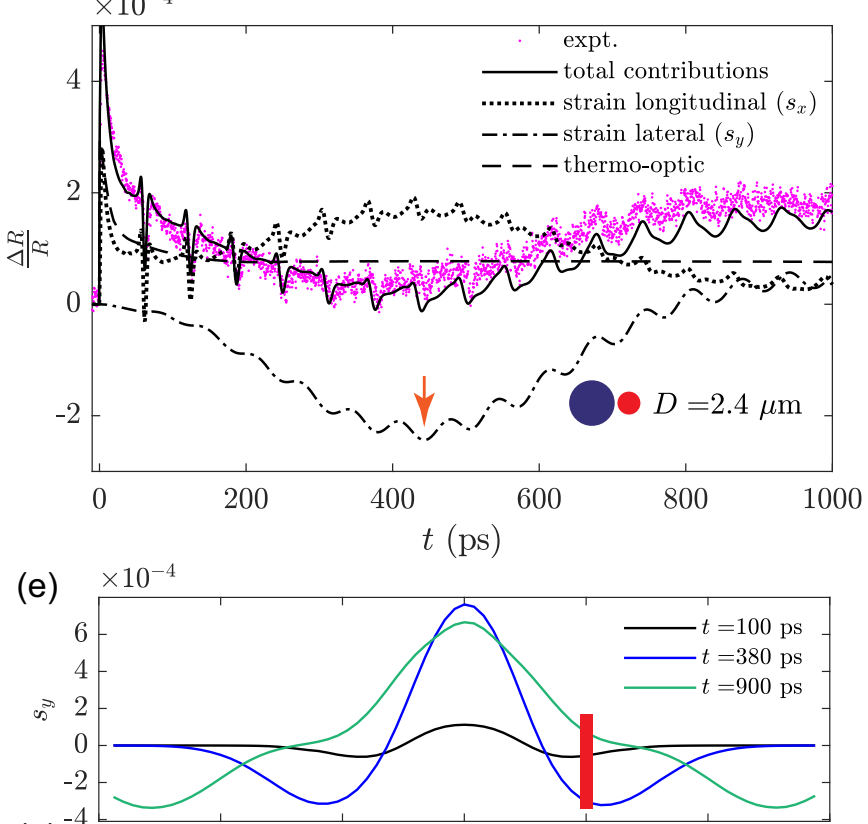

(g)

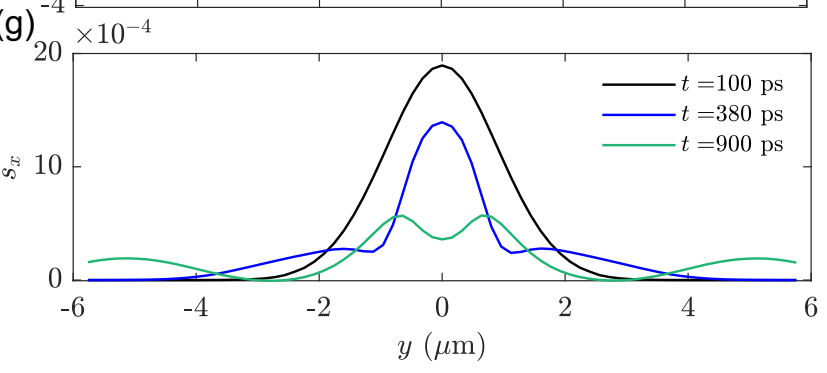

(b)

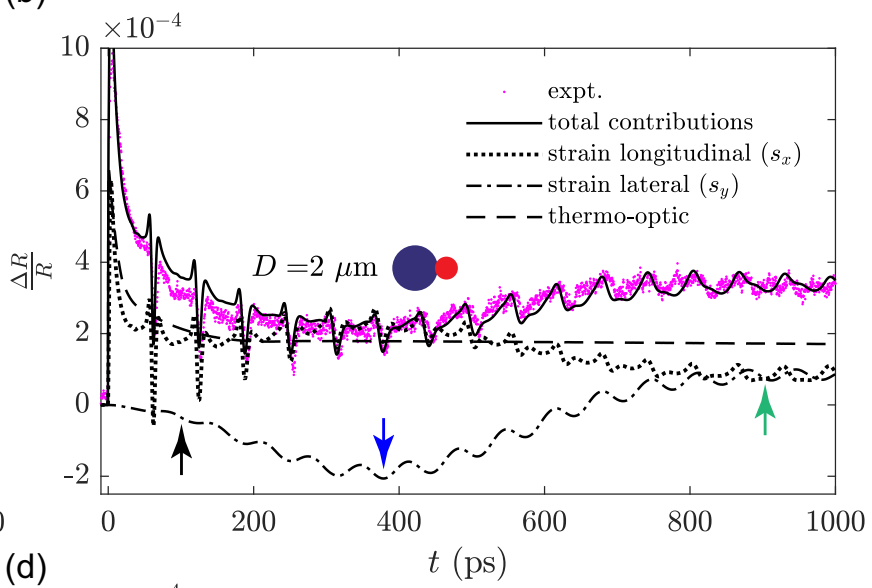

(d)
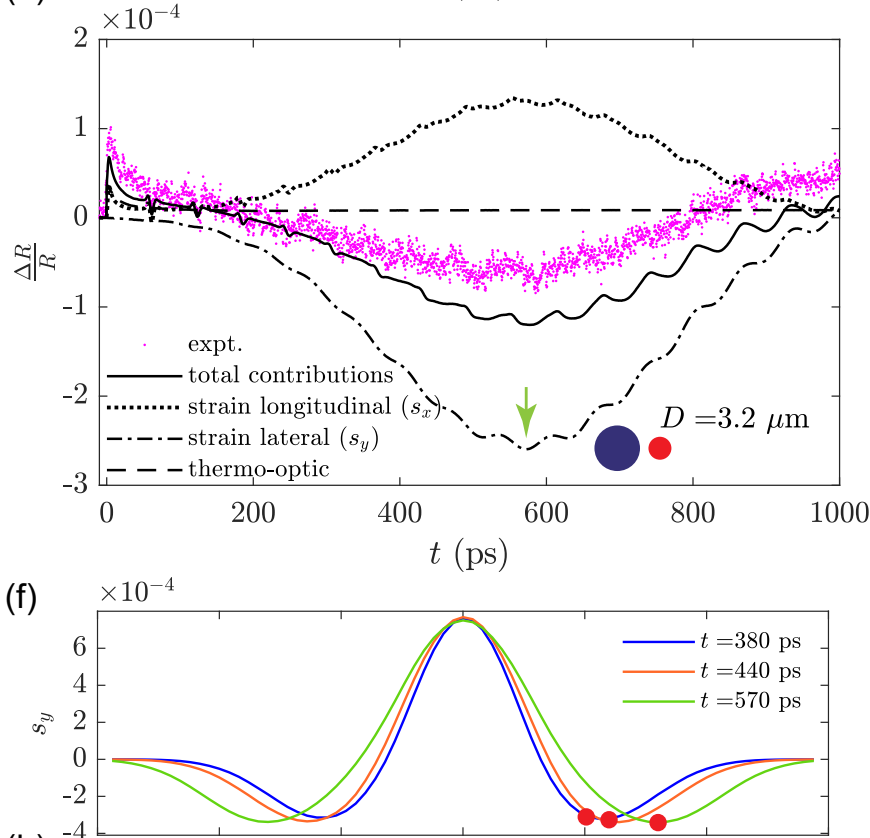

(h)

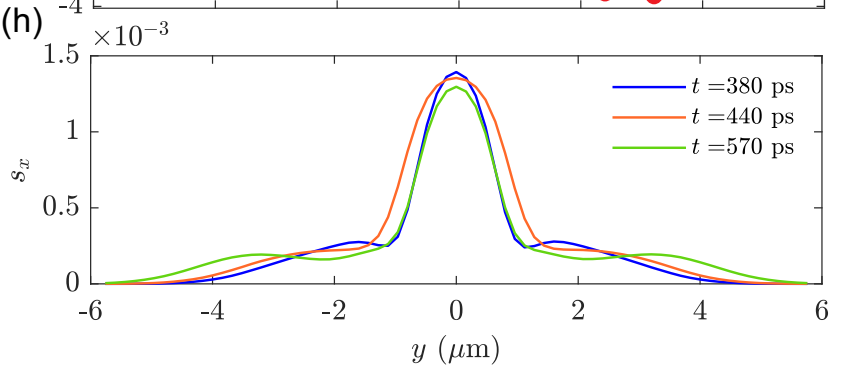

FIG. 3. Measured reflectivity change (magenta dots) and model calculated reflectivity change (black solid lines) at (a) 0, (b) 2, (c) 2.4, and (d) $3.2 \mu \mathrm{m}$ probing positions. The dotted, dash-dotted, and dashed lines are reflectivity changes from each contribution: the longitudinal strain $\left(s_{x}\right)$ contribution, the lateral strain $\left(s_{y}\right)$ contribution, and the lattice temperature contribution (thermo-optic). (e)-(h) The strain profiles along the membrane surface at multiple time delays, separated in the strain components (e),(f) $s_{y}$ and (g),(h) $s_{x}$. The displayed time delays correspond to the colored arrows in (b)-(d). The red bar in (e) illustrates the probing position of $2 \mu \mathrm{m}$. The red filled circles in (f) illustrate the three probing positions. See, also, Video 1 in the Supplemental Material [40].

a thermoreflectance that deviates from its room-temperature value. The effect of this steady-state temperature rise on our signals can be seen at the negative delay times, which are around $1.2 \times 10^{-3}$. We note that this steady-state signal only introduces a constant offset to our data, which does not affect the acoustic dynamics. To compare with the model, this offset is subtracted from the total signal.
The signal after $50 \mathrm{ps}$ is dominated by acoustic dynamics. The thermo-optic effect and the nonpropagating strain contribution are still present, but they contribute to the measured signal in the form of a quasi-DC background. The slope of this background is determined by long-term heat dissipation and relaxation of the surface strain. The repetitive dips with a period of $\sim 64$ ps are caused by the propagation and internal 
reflection of the longitudinal strain component $s_{x}$. The period of these longitudinal echoes is equal to one round-trip time of the acoustic pulse. In Fig. 3(a), we see the broadening and damping of the longitudinal echoes. This is caused by the combined effect of acoustic attenuation and diffraction. In the model, we assume a frequency-dependent acoustic attenuation which is proportional to the square of the acoustic frequency [6]: $\alpha_{l}(\omega)=\alpha_{l 0} \omega^{2}$. The attenuation coefficient $\alpha_{l 0}$ is treated as a fitting parameter, which yields the value $\alpha_{l 0}=$ $3.51 \mathrm{GHz}^{-2} \mathrm{~m}^{-1}$. The quadratic dependence indicates more attenuation for higher frequencies, effectively broadening the acoustic pulse. At this stage, we cannot completely rule out the possibility of interface scattering loss to the attenuation of the acoustic pulse. However, the microscopic roughness at the metal/air interface is expected to be much finer than the acoustic wavelength, and thus scattering loss at our frequency range is expected to be small. The diffraction of the longitudinal acoustic wave also broadens the echo. The acoustic diffraction produces off-axis propagation with a slight angle, which effectively dissipates the energy and elongates the echo.

A striking aspect is the increase in the contribution of the lateral strain component $\left(s_{y}\right)$, which, at zero probing position, gradually reaches its maximum at $\sim 600 \mathrm{ps}$. The tightly focused pump beam means that the effect of acoustic diffraction and the redistribution of acoustic energy from longitudinal strain $\left(s_{x}\right)$ to lateral strain $\left(s_{y}\right)$ significantly changes the signal shape and thus can be measured. If the contribution of $s_{y}$ only slightly changes the signal shape measured at zero probing position, its contribution becomes increasingly important and dominates the overall background shape at large off-center probing positions. In Figs. 3(b)-3(d), we plot the measured signal at three off-center probing positions and the separation of contributions by the model calculations. One interesting observation is the initial decrease and the follow-up increase of the background signal, forming broad dips for these off-center positions. By looking at the $s_{x}$ contribution, such a broad dip is absent for $D=2 \mu \mathrm{m}$. For $D=2.4$ and $D=3.2 \mu \mathrm{m}$, the $s_{x}$ contribution does show a broad peak at the correct timing; however, it is of the opposite sign as the measurements. On the contrary, the $s_{y}$ contribution remarkably reproduces both the timing and the sign of these broad dips. The $s_{y}$ contribution even goes to negative and back to positive again. The off-center probe measures the "passing by" of the Lamb waves traveling in the lateral direction. In Figs. 3(e) and 3(f), we show the lateral strain $\left(s_{y}\right)$ profiles along the surface of the membrane at selected time instants corresponding to the center of these broad dips. At $t=100 \mathrm{ps}$, the surface wave is just generated at the edges of the pump spot. At later times, the surface wave is fully formed and starts to propagate outward from the pumped region. The leading part of this wave is negative in $s_{y}$, while positive in its trailing part. The off-center probing positions therefore undergo relatively slow compressions, followed by expansions caused by the passing by of the lateral strain component. In Figs. 3(g) and 3(h), we show the longitudinal strain $\left(s_{x}\right)$ profiles at the same time instants. The $s_{x}$ at these timings are all positive, and partially cancel out the reflectivity changes from the $s_{y}$ component. The slight disagreement in the amplitude of the broad dip in Fig. 3(d) is expected to be caused by the geometrical diverging effect. For a diverging guided (propagating) acoustic wave spreading from a point source in a two-dimensional space as in the experiment, the wave energy is spread out over the cylindrical surface area $2 \pi r h$, where $r$ is the distance from the wave front to the point source and $h$ is the membrane thickness. Because of energy conservation, this leads to a $1 / r$ decay rate for the wave intensity. Since, for a propagating acoustic wave, the intensity is proportional to the wave amplitude squared [41,42], it translates to a $1 / \sqrt{r}$ law for the wave amplitude. This geometrical diverging effect is absent in the 2D simulations. The diverging effect can be ignored inside the source region, the size of which can be estimated as equal to our focused pump beam size $\left(\approx 2 w_{\text {pump }}=3.6 \mu \mathrm{m}\right)$. For probing positions within this range, especially for Figs. 3(a)-3(c), the diverging effect is minimum and can be neglected.

Figures 4(a) and 4(c) show the measured and the calculated reflectivity changes at all probing positions. These calculations are obtained by using the same best-fit parameters that were determined earlier. The magnitude, evolution of the acoustic echoes, as well as changes in the background signal are well reproduced by the simulations. In Figs. 4(b) and 4(d), the reflectivity changes at all probing positions and time delays are plotted as two-dimensional color plots. In both the measured and calculated data, a white band with oblique orientation is clearly observed (marked by red dashed lines). This white band is a result of lateral propagation of one of the Lamb wave modes, which, owing to the $s_{y}$ strain component, decreases the reflectivity as the Lamb wave passes by the probing position. This is illustrated in Fig. 4(f), where the contribution to the reflectivity due to the $s_{y}$ component is separated. Its negative contribution to the reflectivity and its lateral propagation are clearly seen. From the slopes of the white band in Figs. 4(b) and 4(d), a very similar lateral propagation speed of $\sim 5600 \mathrm{~m} / \mathrm{s}$ is determined. To determine the Lamb wave modes, we retrieve the dispersion relation by a spatiotemporal Fourier transform of the simulated strain field. The results is shown in Fig. 4(e) and compared with the analytical solution of Lamb wave dispersion relations [20,41]. From the dispersion relation, we determine the phase velocity of the $S_{0}$ symmetric mode to be $\sim 5300 \mathrm{~m} / \mathrm{s}$, in good agreement with the retrieved lateral propagation speed from Figs. 4(b) and 4(d). We can also observe that the group velocity of the $S_{0}$ mode is very similar to its phase velocity, suggesting small dispersions for this mode. The agreement on the propagation speed indicates that the broad dips in the signal probed at large off-center positions, and thus the white bands observed in Figs. 4(b) and 4(d), are caused primarily by an excitation of the $S_{0}$ symmetric Lamb mode. The excitation of Lamb waves requires a component of particle movement in the direction parallel to the surface. In our case, this is primarily caused by acoustic diffraction and mode conversion: The tight focusing condition in our experiment results in the generation of a localized acoustic field directly after the excitation. The localization gives rise to a nonzero gradient parallel to the surface in the acoustic field, causing particle movements parallel to the surface because of acoustic diffraction. Another cause of the Lamb wave generation is mode conversion (longitudinal to Lamb modes): Due to acoustic diffraction, part of the longitudinal wave arrives at the surface at an oblique angle, causing particle movement along the surface and converting some of its energy into Lamb 

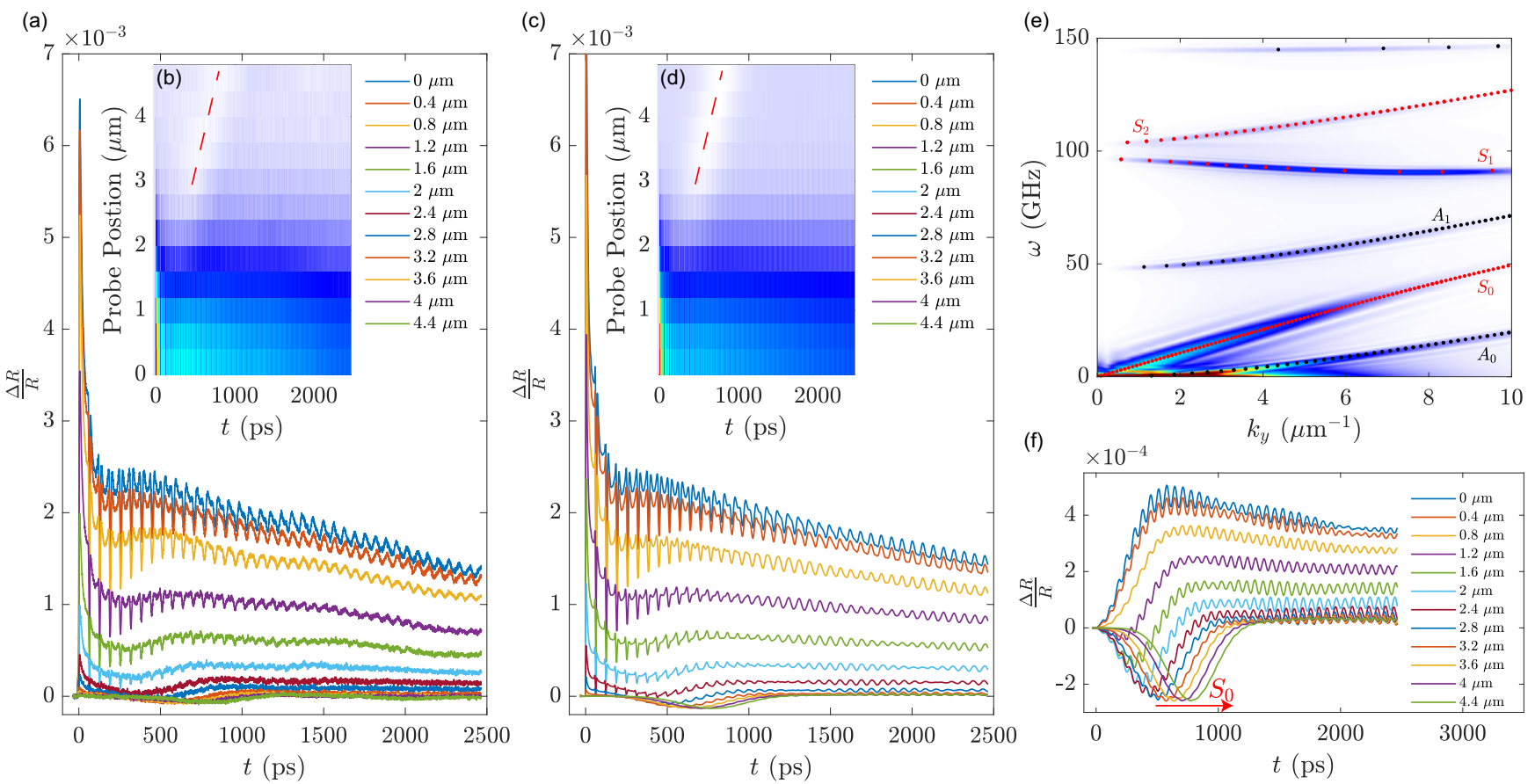

FIG. 4. (a),(b) Measured and (c),(d) calculated reflectivity changes at all probing positions. (b),(d) The red dashed lines highlight the contribution from the lateral propagation of the Lamb waves. Red and green represent higher values, while blue and white represent lower values. (e) The Lamb wave dispersion relation retrieved from the simulation shown as a colored plot. The black and red dots are solutions of the analytical expression of Lamb wave dispersion relations. The black dots correspond to the asymmetric modes and the red dots to the symmetric modes. (f) The calculated reflectivity contribution from the $s_{y}$ component, where the red arrow illustrates the propagation of the $S_{0}$ Lamb mode.

waves [6]. A secondary cause is the nonzero gradient in the thermal stress along the surface direction, which can directly facilitate parallel particle movements. In pump-probe experiments using a conventional geometry, i.e., a large pump spot and a small probe spot, the lateral dynamics or Lamb waves are expected to have negligible or minor contributions to the signals. The gradient in the temperature and the acoustic field in the forward direction is usually quite large in metals due to a shallow light-penetration depth and limited thermal-energy diffusion because of high electron-phonon coupling strength. For the lateral dynamics to significantly change the measured signals, the pump beam needs to be tightly focused. Our simulations with a variety of pump spot sizes show that the lateral dynamics only significantly influences the signal shape when the pump spot radius is smaller than 1 or 2 micrometers.

Several aspects of the data analysis need to be further addressed. First, in the calculations, two aluminum oxide $\left(\mathrm{Al}_{2} \mathrm{O}_{3}\right)$ layers with a thickness of $6 \mathrm{~nm}$ on both sides of the free membrane are included. Ellipsometry measurement on the sample confirms the presence of 6-nm-thick oxide layers. Second, the heat dissipation is expected to be caused by heat diffusion in all lateral directions. However, our 2D simulation results in a slower decay than what is observed in the experiment. To correct for this, we introduced an extra heat-loss term in the lattice temperature equation of the two-temperature model. This term is in the form of $-(1 / \tau)\left[C_{l}\left(T_{l}-T_{0}\right)\right]$, where $C_{l}$ is the lattice heat capacity, $T_{l}$ is the lattice temperature, and $T_{0}=300 \mathrm{~K}$ is the environmental temperature. The parame- ter $\tau$ determines the rate of heat dissipation away from the pumped area and it is treated as a fitting parameter. The best-fit $\tau$ is $5.2 \mathrm{~ns}$. We note that this term does not change the simulated acoustic dynamics. Third, we find that adding acoustic nonlinearity in the calculations significantly improves the agreement between the measured and calculated signals. A comparison between the best-fit results using a linear acoustic model and a nonlinear acoustic model at zero probing position is shown in the inset of Fig. 2. The nonlinear model better reproduces the sawtooth signal shape especially observed at longer time delays. Acoustic nonlinearity is incorporated into the model by adding a second-order term in the strain-stress relation [6]: $\sigma_{x}=-(\lambda+2 \mu) s_{x}-\lambda s_{y}+(\gamma / 4) s_{x}^{2}$. The best-fit nonlinearity constant $\gamma$ is found to be $1.2 \times 10^{13} \mathrm{~Pa}$.

Our 2D nonlinear model shows the formation of sawtoothlike temporal shapes. However, due to the complexity of the model, the point-source excitation, and the combined influence of several physical effects, an intuitive picture explaining the formation of such signal shapes is not readily obtained. To better show the influence of nonlinear effects, we study a simpler system which qualitatively shows the same nonlinear dynamics. Here we simulate the nonlinear propagation of a 1D strain in a $200 \mathrm{~nm}$ aluminium freestanding membrane. The strain pulse is launched in the simulation by a hypothetical source: we assume that the temperature of the surface layer of the membrane is increased by $40 \mathrm{~K}$, with a rising time of $5 \mathrm{ps}$. The surface expansion caused by this swift temperature increase generates a strain pulse propagating into the interior 
(a)

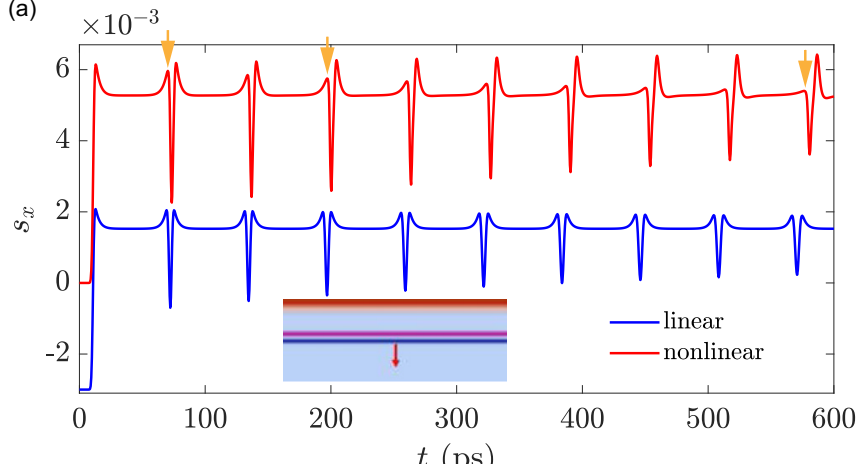

(b) $\times 10^{-3}$

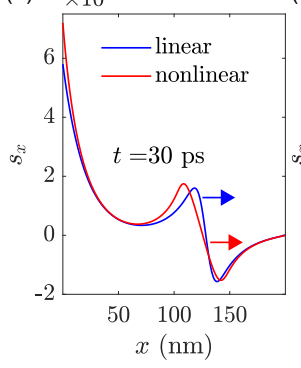

(c) $\times 10^{-3}$

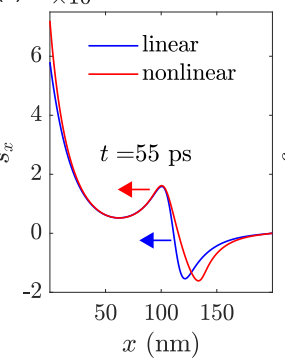

(d) $\times 10^{-3}$

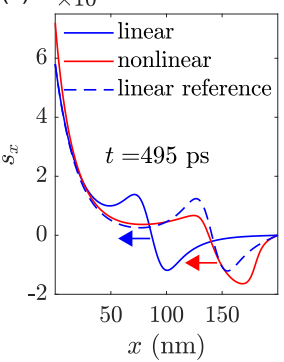

the nonlinear propagation gives rise to increasing asymmetry. As marked by the yellow arrows, the first crest of the echo gradually diminishes, while the second crest seems to grow, as more propagation distance and internal reflections are accumulated. Despite the simplicity of this 1D nonlinear propagation model, the reduction of the first crest can be seen in the experimental data as well, as marked by the yellow arrows in Fig. 2. Because the echoes are not well separated in the actual experiment, the changing heights of these first and second crests give rise to the observed sawtoothlike signals. The asymmetry in the nonlinear echo originates from the asymmetry in the nonlinear strain-stress relation. The sign of the nonlinearity constant determines the direction of this asymmetry, i.e., whether the first or the second crest would become stronger.

These changes in echo shapes result from a straindependent propagation velocity combined with the phase reversal upon reflection at the free surface. From the nonlinear strain-stress relation, we can define an effective speed of sound through $-\rho_{0} c_{\mathrm{eff}}^{2} s_{x}=-\rho_{0} c_{l}^{2} s_{x}+(\gamma / 4) s_{x}^{2}$, which gives

$$
c_{\mathrm{eff}}^{2}=c_{l}^{2}-\frac{\gamma}{4 \rho_{0}} s_{x},
$$

FIG. 5. Nonlinear propagation of a 1D strain pulse. (a) The returned strain at the location close to the membrane front surface ( $5 \mathrm{~nm}$ beneath the surface), as a function of time, for linear (blue line) and nonlinear (red line) propagations. The blue line has a vertical offset of $-3 \times 10^{-3}$ for clarity. The yellow arrows in (a) show the diminishing of the first crest of the echoes in the nonlinear case. The inset in (a) shows a schematic of the simulation scheme, in which the topmost layers of the membrane are heated and a strain pulse is launched into the interior. The red layer illustrates the temperature distribution, while the pink and blue areas illustrate the positive and negative parts of the generated strain. (b)-(d) The strain profiles inside the membrane, at different time instants, for linear (blue lines) and nonlinear (red lines) propagations. The red and blue arrows indicate the propagation directions of the strain pulse. The blue dashed line in (d) is the linear strain offset in position to overlap with the nonlinear strain profile for reference. See, also, Video 2 in the Supplemental Material [40].

of the membrane. A schematic of the simulation scheme is shown in the inset of Fig. 5(a). The temperature in the heated layer follows an exponential decay with a characteristic length of $20 \mathrm{~nm}$. This heat source is much more localized to the membrane surface than that in the experiment. This strongly localized heating ensures a narrow spatial extent of the resulting strain pulse. This gives rise to well-separated echoes and facilitates the observation of propagation-related effects. In Fig. 5(a), we plot the strain $s_{x}$ at $5 \mathrm{~nm}$ below the excitation surface, as a function of time, for both linear and nonlinear propagations. These traces show the time evolutions of the generated strain as it undergoes multiple round trips in the membrane. For the simulation of nonlinear propagation, as mentioned before, an additional second-order term is added to the strain-stress relation: $\sigma_{x}=-(\lambda+2 \mu) s_{x}+(\gamma / 4) s_{x}^{2}$. This relation implies that the atoms in the lattice experience a nonlinear restoring force upon displacement from equilibrium. While symmetric echoes are observed for the linear case,

where $c_{l}$ is the speed of sound in the absent of nonlinearity. The effect of this strain-dependent velocity upon propagation is shown in Figs. 5(b)-5(d). An additional complicating factor is the reflection at free surfaces: Fig. 5(c) shows a strain pulse after reflection at the back surface, where a sign reversal has occurred due to the impedance mismatch between aluminum and air. As a result, the velocity of the leading and trailing parts of the strain profile will have changed after such a reflection, further complicating the profile distortion. The calculation shows a significant reshaping of the propagating strain pulse after several round trips, as shown in Fig. 5(d) by the red trace.

The above analysis of the nonlinear effects is based on a simplified 1D picture. In our experiment, the signal change due to the nonlinear effect may be affected by other factors, such as a larger spatial extent of the generated strain, acoustic diffraction due to the localized excitation volume, the contribution of different acoustic field components, etc. Although it is difficult to separate the effect of each of these contributions on the nonlinear strain propagation, these effects are included in our 2D simulations.

\section{SUMMARY}

Lamb waves are excited in a thin, freestanding aluminum membrane by a tightly focused fs laser beam. The optical response caused by the excitation is measured at a range of probing positions around the excitation region by delayed probe pulses. The measured reflectivity changes at all positions and time delays are compared with the calculations by using a 2D numerical model. Excellent agreements between measurements and calculations were found. This combined approach allows the separation of contributions to the measured signals, as well as the role of different strain components on the signal formations, which have not been clearly elaborated in previous studies. It has been shown that the measured signal is a combination of several contributions: 
the thermo-optic contribution, and the strain-optic contribution due to longitudinal and lateral strains. The off-center probe registers the lateral propagation of the excited Lamb waves, which is identified as the $S_{0}$ symmetric Lamb mode by comparing with simulations. In addition, the excellent model fit to the measured signals allows one to retrieve quantitative information on several parameters, such as the two complex photoelastic coefficients and the acoustic attenuation coefficient. Furthermore, evidence of acoustic nonlinearity in the excited Lamb waves was found and a nonlinearity constant is extracted.

\section{ACKNOWLEDGMENTS}

This research was supported by the European Research Council (ERC Starting Grant No. 637476) and the Netherlands Organization for Scientific Research (NWO). This work was conducted at the Advanced Research Center for Nanolithography, a public-private partnership between the University of Amsterdam, Vrije Universiteit Amsterdam, the Netherlands Organization for Scientific Research (NWO), and the semiconductor-equipment manufacturer ASML.

H.Z. and A.A. contributed equally to this work.
[1] M. Metcalfe, S. M. Carr, A. Muller, G. S. Solomon, and J. Lawall, Resolved Sideband Emission of InAs/GaAs Quantum Dots Strained by Surface Acoustic Waves, Phys. Rev. Lett. 105, 037401 (2010).

[2] K. J. Satzinger, Y. P. Zhong, H. S. Chang, G. A. Peairs, A. Bienfait, M.-H. Chou, A. Y. Cleland, C. R. Conner, E. Dumur, J. Grebel, I. Gutierrez, B. H. November, R. G. Povey, S. J. Whiteley, D. D. Awschalom, D. I. Schuster, and A. N. Cleland, Quantum control of surface acoustic-wave phonons, Nature (London) 563, 661 (2018).

[3] Y. Chu, P. Kharel, W. H. Renninger, L. D. Burkhart, L. Frunzio, P. T. Rakich, and R. J. Schoelkopf, Quantum acoustics with superconducting qubits, Science 358, 199 (2017).

[4] B. C. Daly, N. C. R. Holme, T. Buma, C. Branciard, and T. B. Norris, Imaging nanostructures with coherent phonon pulses, Appl. Phys. Lett. 84, 5180 (2004).

[5] K.-H. Lin, C.-T. Yu, S.-Z. Sun, H.-P. Chen, C.-C. Pan, J.-I. Chyi, S.-W. Huang, P.-C. Li, and C.-K. Sun, Two-dimensional nanoultrasonic imaging by using acoustic nanowaves, Appl. Phys. Lett. 89, 043106 (2006).

[6] H. Zhang, A. Antoncecchi, S. Edward, I. Setija, P. Planken, and S. Witte, Unraveling Phononic, Optoacoustic, and Mechanical Properties of Metals with Light-Driven Hypersound, Phys. Rev. Appl. 13, 014010 (2020).

[7] J. N. Hernandez-Charpak, K. M. Hoogeboom-Pot, Q. Li, T. D. Frazer, J. L. Knobloch, M. Tripp, S. W. King, E. H. Anderson, W. Chao, M. M. Murnane, H. C. Kapteyn, and D. Nardi, Full characterization of the mechanical properties of $11-50 \mathrm{~nm}$ ultrathin films: Influence of network connectivity on the poisson's ratio, Nano Lett. 17, 2178 (2017).

[8] S. Edward, H. Zhang, I. Setija, V. Verrina, A. Antoncecchi, S. Witte, and P. Planken, Detection of Hidden Gratings through Multilayer Nanostructures using Light and Sound, Phys. Rev. Appl. 14, 014015 (2020).

[9] C. Thomsen, H. T. Grahn, H. J. Maris, and J. Tauc, Surface generation and detection of phonons by picosecond light pulses, Phys. Rev. B 34, 4129 (1986).

[10] O. B. Wright, Ultrafast nonequilibrium stress generation in gold and silver, Phys. Rev. B 49, 9985 (1994).

[11] A. Devos and C. Lerouge, Evidence of Laser-Wavelength Effect in Picosecond Ultrasonics: Possible Connection with Interband Transitions, Phys. Rev. Lett. 86, 2669 (2001).

[12] T. F. Crimmins, A. A. Maznev, and K. A. Nelson, Transient grating measurements of picosecond acoustic pulses in metal films, Appl. Phys. Lett. 74, 1344 (1999).
[13] T. Saito, O. Matsuda, and O. B. Wright, Picosecond acoustic phonon pulse generation in nickel and chromium, Phys. Rev. B 67, 205421 (2003).

[14] P. J. S. van Capel and J. I. Dijkhuis, Time-resolved interferometric detection of ultrashort strain solitons in sapphire, Phys. Rev. B 81, 144106 (2010).

[15] O. Matsuda, O. B. Wright, D. H. Hurley, V. Gusev, and K. Shimizu, Coherent shear phonon generation and detection with picosecond laser acoustics, Phys. Rev. B 77, 224110 (2008).

[16] C. Rossignol, J. M. Rampnoux, M. Perton, B. Audoin, and S. Dilhaire, Generation and Detection of Shear Acoustic Waves in Metal Submicrometric Films with Ultrashort Laser Pulses, Phys. Rev. Lett. 94, 166106 (2005).

[17] B. Giammarinaro, D. Espindola, F. Coulouvrat, and G. Pinton, Focusing of Shear Shock Waves, Phys. Rev. Appl. 9, 014011 (2018).

[18] J. J. Kasinski, L. Gomez-Jahn, K. J. Leong, S. M. Gracewski, and R. J. D. Miller, Optical generation of coherent surface acoustics: An optically based probe of surface structure and dynamics, Opt. Lett. 13, 710 (1988).

[19] Q. Li, K. Hoogeboom-Pot, D. Nardi, M. M. Murnane, H. C. Kapteyn, M. E. Siemens, E. H. Anderson, O. Hellwig, E. Dobisz, B. Gurney, R. Yang, and K. A. Nelson, Generation and control of ultrashort-wavelength two-dimensional surface acoustic waves at nanoscale interfaces, Phys. Rev. B 85, 195431 (2012).

[20] H. Lamb, On waves in an elastic plate, Proc. R. Soc. London A 93, 114 (1917).

[21] Q. Xie, S. Mezil, P. H. Otsuka, M. Tomoda, J. Laurent, O. Matsuda, Z. Shen, and O. B. Wright, Imaging gigahertz zerogroup-velocity lamb waves, Nat. Commun. 10, 2228 (2019).

[22] S. Mezil, F. Bruno, S. Raetz, J. Laurent, and D. Royer, Investigation of interfacial stiffnesses of a tri-layer using zero-group velocity Lamb modes, J. Acoust. Soc. Am. 138, 3202 (2015).

[23] G. Yan, S. Raetz, N. Chigarev, V. E. Gusev, and V. Tournat, Characterization of Progressive Fatigue Damage in Solid Plates by Laser Ultrasonic Monitoring of Zero-Group-Velocity Lamb Modes, Phys. Rev. Appl. 9, 061001(R) (2018).

[24] D. M. Photiadis, M. K. Zalalutdinov, A. S. Bracker, S. G. Carter, D. Gammon, and B. H. Houston, Photoexcited elastic waves in freestanding GaAs films, Phys. Rev. B 101, 245304 (2020).

[25] M. Grossmann, O. Ristow, M. Hettich, C. He, R. Waitz, E. Scheer, V. Gusev, T. Dekorsy, and M. Schubert, Time-resolved detection of propagating Lamb waves in thin silicon membranes 
with frequencies up to $197 \mathrm{GHz}$, Appl. Phys. Lett. 106, 171904 (2015).

[26] T. Tachizaki, T. Muroya, O. Matsuda, Y. Sugawara, D. H. Hurley, and O. B. Wright, Scanning ultrafast Sagnac interferometry for imaging two-dimensional surface wave propagation, Rev. Sci. Instrum. 77, 043713 (2006).

[27] S. I. Anisimov, B. L. Kapeliovich, and T. L. Perel'man, Electron emission from metal surfaces exposed to ultrashort laser pulses, Sov. Phys.-JETP 39, 375 (1974).

[28] P. M. Shearer, Introduction to Seismology (Cambridge University Press, New York, 2009).

[29] R. D. Blandford and K. S. Thorne, Modern Classical Physics: Optics, Fluids, Plasmas, Elasticity, Relativity, and Statistical Physics (Princeton University Press, Princeton, NJ, 2017).

[30] K. Yee, Numerical solution of initial boundary value problems involving Maxwell's equations in isotropic media, IEEE Trans. Antennas Propag. 14, 302 (1966).

[31] D. M. Sullivan, Electromagnetic Simulation using the FDTD Method (IEEE Press, New Jersey, 2000).

[32] M. Born and E. Wolf, Principles of Optics (Cambridge University Press, Cambridge, 1999).

[33] D. R. Lide, CRC Handbook of Chemistry and Physics, Internet Version 2005 (CRC Press, Boca Raton, FL, 2005).

[34] Z. Lin, L. V. Zhigilei, and V. Celli, Electron-phonon coupling and electron heat capacity of metals under conditions of strong electron-phonon nonequilibrium, Phys. Rev. B 77, 075133 (2008).

[35] T. Dehoux, N. Chigarev, C. Rossignol, and B. Audoin, Three-dimensional elasto-optical interaction for reflectometric detection of diffracted acoustic fields in picosecond ultrasonics, Phys. Rev. B 76, 024311 (2007).

[36] T.Favaloro, J.-H. Bahk, and A. Shakouri, Characterization of the temperature dependence of the thermoreflectance coefficient for conductive thin films, Rev. Sci. Instrum. 86, 024903 (2015).

[37] Y. Wang, J. Y. Park, Y. K. Koh, and D. G. Cahill, Thermoreflectance of metal transducers for time-domain thermoreflectance, J. Appl. Phys. 108, 043507 (2010).

[38] R. B. Wilson, B. A. Apgar, L. W. Martin, and D. G. Cahill, Thermoreflectance of metal transducers for optical pump-probe studies of thermal properties, Opt. Express 20, 28829 (2012).

[39] A. G. Mathewson and H. P. Myers, Optical absorption in aluminium and the effect of temperature, J. Phys. F: Metal Phys. 2, 403 (1972).

[40] See Supplemental Material at http://link.aps.org/supplemental/ 10.1103/PhysRevB.103.064303 for videos of calculated displacement vector and strain.

[41] J. L. Rose, Ultrasonic Guided Waves in Solid Media (Cambridge University Press, New York, 2014).

[42] B. Lüthi, Physical Acoustics in the Solid State (Springer, Berlin, 2005). 